\title{
UNIFORM BOUNDS FOR LIMITED SETS AND APPLICATIONS TO BOUNDING SETS.
}

\author{
BENGT JOSEFSON
}

\begin{abstract}
A set $D$ in a Banach space $E$ is limited if $\limsup _{k \rightarrow \infty} \sup _{z \in D}\left|\varphi_{k}(z)\right|>0 \Rightarrow \sup _{\|z\|=1} \lim \sup _{k \rightarrow \infty}$ $\left|\varphi_{k}(z)\right|>0$ for every sequence $\left(\varphi_{k}\right) \subset E^{\star}$. It is studied how this implication can be quantified, for example if there exists a constant $C>0$ such that $\limsup _{k \rightarrow \infty} \sup _{z \in D}\left|\varphi_{k}(z)\right|=1 \Rightarrow$ $\sup _{\|z\|=1} \lim \sup _{k \rightarrow \infty}\left|\varphi_{k}(z)\right| \geq C$ for every sequence $\left(\varphi_{k}\right) \subset E^{\star}$, is studied. Relatively compact sets and limited sets in $l^{\infty}$ - among others the unit vectors - have uniform bounds in this sense. A fundamental example of a limited set without any uniform bounds is constructed. A set $D$ is called bounding if $f(D)$ is bounded for every entire function on $E$. That bounding sets are uniformly limited and that strongly bounding sets are limited in the strongest sense are proved. Examples show that the convex hull of bounding sets in general are not bounding and that bounding sets in general does not have Grothendieck's incapsulating property as relatively weakly compact sets have.
\end{abstract}

\section{Introduction}

The Banach spaces considered here are over the the real or the complex field.

A set $D$ in a Banach space $E$ is called limited if every weak ${ }^{\star}$ null sequence $\left(\varphi_{k}\right)$ in the dual space $E^{\star}$ converges uniformly on $D$, i.e.

$$
\lim _{k \rightarrow \infty} \sup _{z \in D}\left|\varphi_{k}(z)\right|=0
$$

An equivalent formulation without referring to null sequences is: $D$ is called limited if

$$
\limsup _{k \rightarrow \infty} \sup _{z \in D}\left|\varphi_{k}(z)\right|>0 \Rightarrow \sup _{\|z\|=1} \limsup _{k \rightarrow \infty}\left|\varphi_{k}(z)\right|>0
$$

for every sequence $\left(\varphi_{k}\right) \subset E^{\star}$.

A natural question is how this relation can be quantified. For example we may ask if a limited set $D$ has a constant $C>0$ such that

$$
\limsup _{k \rightarrow \infty} \sup _{z \in D}\left|\varphi_{k}(z)\right|=1 \Rightarrow \sup _{\|z\|=1} \limsup _{k \rightarrow \infty}\left|\varphi_{k}(z)\right| \geq C
$$

for every sequence $\left(\varphi_{k}\right) \subset E^{\star}$. Such a limited set is called limited in the 
strongest sense (the class $\mathscr{L}_{1}$ ). Relatively compact sets and limited sets in $l^{\infty}$ - among others the unit vectors - are uniformly limited in this sense (see remark 6).

Four different notions of uniform bounds are introduced. The weakest one, called uniformly limited (the class $\mathscr{L}_{4}$ in Definition 2), is a sequential property (such as being compact or limited) in contrast to being in $\mathscr{L}_{1}$.

Recall that a set $D \subset E$ is bounding if $f(D)$ is bounded for every entire function $f: E \rightarrow \mathrm{C}$ and that $f(z)=\sum_{k=0}^{\infty} \varphi_{k}^{k}(z)$ is an entire function if and only if $\left(\varphi_{k}\right)_{k} \subset E^{\star}$ is a weak ${ }^{\star}$ null sequence. Thus compact sets are bounding which in turn are limited and, in Banach spaces without any copy of $l_{1}$, limited sets are weakly compact according to [1]. Theorem 2 sharpens this wellknown inclusion to say that bounding sets are uniformly limited.

That this is a sharpening follows from Theorem 1. A Banach space $E$ containing an isometric copy $\left(a_{k}\right)$ of the unit vectors of $c_{0}$ as a limited set is constructed such that for every $\varepsilon>0$, there exists $\left(\varphi_{k}\right) \subset E^{\star}$ with $\left\|\varphi_{k}\right\|=1=\varphi_{k}\left(a_{k}\right)$, for every $k$, but $\sup _{\|z\|=1} \lim \sup _{k \rightarrow \infty}\left|\varphi_{k}(z)\right| \leq \varepsilon$, denying any possible uniform bound. Of course this gives a new example of a limited set which is not bounding. Other examples can be found in [6] and [7].

A set $D \subset E$ is called strongly bounding (the class $\mathscr{B}_{s}$ ) if $f(D)$ is bounded for every function holomorphic in some ball $r B_{E}$ with $r>\sup _{z \in D}\|z\|$, where $B_{E}$ denotes the open unit ball of $E$. Relatively compact sets are obviously strongly bounding and, according to [5], so is also every limited set in $l^{\infty}$. Theorem 3 proves that strongly bounding sets in fact are limited in the strongest sense.

In the other direction Theorem 4 shows that a strongly uniformly limited set is bounding for holomorphic functions generated by uniformly bounded linear functionals (the class $\mathscr{B}_{l c}$ in Definition 4). Examples show that Theorem 4 cannot be essentially improved.

Section 2 treats the class of bounding sets $(\mathscr{B})$ and the different classes of limited sets regarding two basic properties - being closed under taking convex hulls (the H-property for short) and having Grothendieck's incapsulating property (the G-property), i.e. $D$ is in the class if (and only if) there is, for every $\epsilon>0$, a set $D_{\epsilon}$ in the class such that $D \subset D_{\epsilon}+\epsilon B_{E}$. In [8] this analogy of Grothendieck's characterization of the relatively weakly compact sets is observed to hold for limited sets. It is easily verified that the classes of compact, limited and uniformly limited sets have both properties. On the contrary $\mathscr{L}_{1}$ fails the G-property while the class of bounding sets fails both of them. The question whether the convex hull of a bounding set also is bounding has been open for quite a while and the negative answer underlines the complexity of bounding sets.

Altogether the following scheme, (see Definitions 1-4), is proved: 


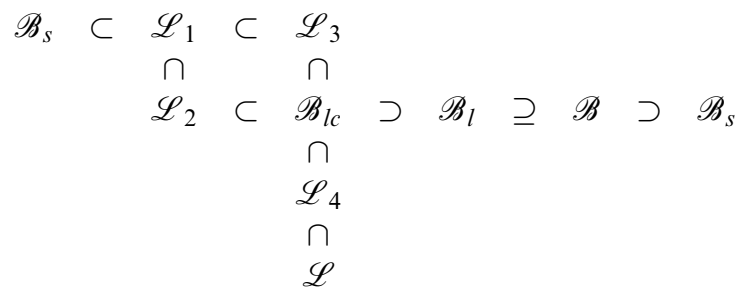

We do not know if the inclusion $\mathscr{B} \subseteq \mathscr{B}_{l}$ is strict or not, but otherwise the inclusions are strict. The scheme is also complete in the sense that if it does not give any information about two classes - there exist no inclusions between them. For example there are sets in $\mathscr{B}$ which are not in $\mathscr{L}_{1}$ and sets in $\mathscr{L}_{1}$ which are not bounding.

Through a number of examples there is a common theme in the constructions. As a starting-point serves $c_{0}$ and the key point is to split each unit vector into parts to give an inner structure. These new units are then connected by $l^{\infty}$ and $l_{1}$ structures. Besides Example 4 only linear functionals are considered.

\section{Uniform bounds}

Definition 1. A set $D \subset E$ is called limited in the strongest sense if there exists a constant $C>0$ such that

$$
\limsup _{k \rightarrow \infty} \sup _{z \in D}\left|\varphi_{k}(z)\right|=1 \Rightarrow \sup _{\|z\|=1} \limsup _{k \rightarrow \infty}\left|\varphi_{k}(z)\right| \geq C
$$

for every sequence $\left(\varphi_{k}\right) \subset E^{\star}$.

Further $D$ is said to be limited in the strong sense if there exists, to every $K>0$, a constant $C_{K}>0$ such that

$$
\limsup _{k \rightarrow \infty} \sup _{z \in D}\left|\varphi_{k}(z)\right|=1 \Rightarrow \sup _{\|z\|=1} \limsup _{k \rightarrow \infty}\left|\varphi_{k}(z)\right| \geq C_{K}
$$

for every sequence $\left(\varphi_{k}\right) \subset E^{\star}$ norm-bounded by $K$ (i.e. $\sup _{k}\left\|\varphi_{k}\right\| \leq K$ ).

The classes of sets limited in the strongest respectively the strong sense are denoted $\mathscr{L}_{1}$ respectively $\mathscr{L}_{2}$.

REMARK 1. A relatively compact set $D$ is limited in the strongest sense because every sequence in $D$ has a clusterpoint and we may take $C=1$. In $l^{\infty}$ all limited sets are limited in the strongest sense according to Remark 6 below. In particular the unit vectors and the unit ball of $c_{0}$ are in $\mathscr{L}_{1}$ with $C=1$.

We shall first construct an example showing that not all limited sets are in the class $\mathscr{L}_{2}\left(\supset \mathscr{L}_{1}\right)$.

We need Banach spaces with the Gelfand-Phillips property (GP-spaces), 
i.e. limited sets are relatively compact, but demanding weak ${ }^{\star}$ null sequences with big norms to deny the limitedness of isometric copies of the unit vectors of $c_{0}$. Such spaces are an essential part of the construction in [6]. We recall the construction with some modifications.

Let $M \subset \mathrm{N}$ be infinite and let $P_{\infty}(M)$ denote the set of all infinite subsets of $M$. Put $\Gamma=P_{\infty}(\mathrm{N}) \times \omega_{1}$, where $\omega_{1}$ denotes the first uncountable ordinal. For each infinite $M \subset \mathrm{N}$, choose a family $\left\{V_{M, \alpha}: \alpha<\omega_{1}\right\} \subset P_{\infty}(M)$ satisfying

$$
V_{M, \alpha} \cap V_{M, \beta} \text { is finite if } \alpha \neq \beta .
$$

Keep $n \in \mathrm{N}$ fixed. Let $\left(a_{(k, n)}\right)_{k}$ be the unit vectors for some $l^{\infty}\left(S_{n}\right)$. Put $x_{(M, \alpha, n)}^{\prime}=\sum_{k \in V_{M, \alpha}} a_{(k, n)}$. Let $\left\{y_{\lambda}: \lambda \in \Lambda_{n}\right\} \subset l^{\infty}\left(S_{n}\right)$ be a set of vectors containing a Hamel-basis of norm one vectors for $l^{\infty}\left(S_{n}\right)$ and the set $\left\{x_{(M, \alpha, n)}^{\prime}:(M, \alpha) \in \Gamma\right\}$

Let $\left\{f_{(\lambda, n)}: \lambda \in \Lambda_{n}\right\}$ be the unit vectors of some $l_{1}\left(\Lambda_{n}\right)$, which we view as a subspace of some $l^{\infty}\left(S_{n}^{\prime}\right)$, and put $z_{\lambda}=y_{\lambda}+\frac{1}{n^{2}} f_{(\lambda, n)}$. Note that $(M, \alpha, n) \in \Lambda_{n}$ for all $(M, \alpha) \in \Gamma$. Especially we put $x_{(M, \alpha, n)}=x_{(M, \alpha, n)}^{\prime}+\frac{1}{n^{2}} f_{(M, \alpha, n)}$. Denote by $F_{n}$ the Banach space, isometric to $c_{0}$, generated by $\left\{a_{(k, n)}: k \in \mathrm{N}\right\}$. Let $E_{n}^{\prime} \subset l^{\infty}\left(A_{n}\right)$, where $A_{n}=S_{n} \cup S_{n}^{\prime}$, be the Banach space generated by $\left\{z_{\lambda}: \lambda \in \Lambda_{n}\right\}$. Let $E_{n}$ be the same vector space but with the equivalent norm

$$
\|\|=\max \left[\|\|_{l^{\infty}\left(A_{n}\right)}, n\|\|_{l^{\infty}\left(A_{n}\right) / F_{n}}\right]
$$

Note that $\left\|x_{(M, \alpha, n)}\right\|=n$ but $\left\|a_{(k, n)}\right\|=1$ for all $(M, \alpha) \in \Gamma$ and all $k$. Note also that the quotient space $E_{n} / F_{n}$ is isomorphic to $l_{1}\left(\Lambda_{n}\right)$, because of the terms $\left\{f_{(\lambda, n)}: \lambda \in \Lambda_{n}\right\}$.

Lemma 1. The Banach space $E_{n}$ has the Gelfand-Phillips property. If $\left(\varphi_{k}\right)_{k}$ is a weak $^{\star}$ null sequence in $E_{n}^{\star}$ with $\lim \sup _{k \rightarrow \infty}\left|\varphi_{k}\left(a_{(k, n)}\right)\right|=1$, then $\lim \sup _{k \rightarrow \infty}\left\|\varphi_{k}\right\| \geq n / 4$.

Proof. The proof is similar to that of Lemma 1 in [6]. Since $E_{n} / F_{n}$ is isomorphic to $l_{1}\left(\Lambda_{n}\right)$, there exists a projection $P: E_{n} \rightarrow F_{n}$. Both $l_{1}\left(\Lambda_{n}\right)$ and $F_{n}$ (isometric to $c_{0}$ ) are GP-spaces. Thus $D$ limited in $E_{n}$ implies that both $D / F_{n}$ and $P(D)$ are relatively compact and hence $D$ is relatively compact, which proves that $E$ has the GP-property.

Assume that $\left(\varphi_{k}\right)$ is a weak ${ }^{\star}$ null sequence in $E_{n}^{\star}$ with $\left\|\varphi_{k}\right\|<n / 4$ and

$$
\limsup _{k \rightarrow \infty}\left|\varphi_{k}\left(a_{(k, n)}\right)\right|=1 \text {. }
$$

Since

$$
\lim _{k \rightarrow \infty} \varphi_{k}\left(a_{(j, n)}\right)=0=\lim _{j \rightarrow \infty} \varphi_{k}\left(a_{(j, n)}\right)
$$


and $\left(a_{(k, n)}\right)$ is isometric to the unit vectors of $c_{0}$, a standard perturbation and subsequence argument shows that we may assume, that for an infinite set $M$, we have $\varphi_{k}\left(a_{(k, n)}\right)=1$ but $\varphi_{k}\left(a_{(j, n)}\right)=0$ when $k \neq j$ and $k, j \in M$.

Keep $\alpha<\omega_{1}$ fixed for a moment. Since

$$
\lim _{k \rightarrow \infty} \varphi_{k}\left(x_{(M, \alpha, n)}\right)=0
$$

there exists $U_{M, \alpha} \subset V_{M, \alpha}$ such that $V_{M, \alpha} \backslash U_{M, \alpha}$ is finite and such that $\left|\varphi_{k}\left(x_{(M, \alpha, n)}\right)\right|<1 / n$ for all $k \in U_{M, \alpha}$.

Since $\omega_{1}$ is uncountable, there exist $\alpha_{1}, \ldots, \alpha_{n^{2}}$ and $j$ such that

$$
j \in \bigcap_{l=1}^{n^{2}} U_{M, \alpha_{l}}
$$

Put

$$
x=\frac{1}{n^{2}} \sum_{l=1}^{n^{2}} x_{\left(M, \alpha_{l}, n\right)}=a_{(j, n)}+z
$$

Since $V_{M, \alpha} \cap V_{M, \beta}$ is finite if $\alpha \neq \beta$ we have that

$$
\frac{1}{n^{2}}\left\|\sum_{l=1}^{n^{2}} x_{\left(M, \alpha_{l}, n\right)}\right\|_{E_{n} / F_{n}}=1 / n
$$

according to 1$)$. Since also $\left\|\frac{1}{n^{2}} \sum_{l=1}^{n^{2}} \frac{1}{n^{2}} f_{\left(M, \alpha_{l}, n\right)}\right\|_{l^{\infty}\left(A_{n}\right)}=1 / n^{2}$ we conclude that $\|z\|_{E_{n} / F_{n}}=1 / n$. Note also that $\|z\|=\|z\|_{E_{n} /\left[a_{(j, n)}\right]}$, where $\left[a_{(j, n)}\right]$ is the subspace generated by $a_{(j, n)}$. Hence

$$
1 / n>\left|\varphi_{j}(x)\right| \geq\left|\varphi_{j}\left(a_{(j, n)}\right)\right|-\left|\varphi_{j}(z)\right|>1-\frac{n}{4} \frac{1}{n}=3 / 4
$$

which gives a contradiction if $n>1$ and completes the proof.

Remark 2. In [4] and [8] it is observed that $E$ is a GP-space if the dual ball $B_{1}$ contains a sequentially weak ${ }^{\star}$-precompact $E$-norming subset $B$, i.e., there exists $c>0$ so that $\|z\| \leq c \sup _{\varphi \in B}|\varphi(z)|$ for every $z \in E$. A direct proof of this gives that if $D=\left\{e_{n}\right\} \subset E$ satisfies $\left\|e_{n}\right\| \geq d$ and $d \leq\left\|e_{n}-e_{j}\right\|$ for a given $d>0$ and all $n \neq j$, then there exists a weak ${ }^{\star}$ null sequence $\left(\varphi_{n}\right) \subset 8 c B_{1}$ with $\lim \sup \left|\varphi_{n}\left(e_{n}\right)\right| \geq d$. (For given infinite $U$ and given $k>l$ there exist $\psi \in B$ and an infinite $V \subset U$ such that $\left|\psi\left(e_{k}-e_{l}\right)\right| \geq d / c$ and such that $\lim _{n \rightarrow \infty, n \in V} \psi\left(e_{n}\right)$ exists. Thus there exist a weak ${ }^{\star}$ Cauchy sequence $\left(\psi_{k}\right) \subset B$ and $\left(j_{k}\right)$ such that $\left|\psi_{k}\left(e_{j_{2 k}}-e_{j_{2 k-1}}\right)\right| \geq d / c$ but $\left|\psi_{k}\left(e_{j_{m}}-e_{j_{n}}\right)\right| \leq d / 2 c$ if $m \geq n>2 k$. Hence $\left(\varphi_{k}\right)=\left(\psi_{k}-\psi_{k-1}\right)$ is a weak ${ }^{\star}$ null sequence in $E^{\star}$, $\left\|\varphi_{k}\right\| \leq 2$ and $\left|\varphi_{k}\left(e_{j_{i}}\right)\right| \geq d / 4 c$ for $i=2 k-1$ or $i=2 k$.) 
On the other hand Theorem 10 in [8] gives that if $X=\left(\oplus_{k \in \mathrm{N}} X_{k}\right)_{l_{1}}$, where $X_{k}$ are GP-spaces such that every sequence $\left(c_{k}\right)_{k}$ of norming constants for $X_{k}$ is unbounded, then $X$ is a GP-space without a sequentially weak ${ }^{\star}$-precompact, $X$-norming subset of the dual ball. A sequence $\left(X_{k}\right)$ with these properties is also constructed in [8]. According to the Lemma $X=$ $\left(\oplus_{n \in \mathrm{N}} E_{n}\right)_{l_{1}}$ is another example of this kind. (The results of Schlumprecht appeared already in [9].)

Remark 3. Without the terms $\left\{f_{(\lambda, n)}: \lambda \in \Lambda\right\}$ in the construction, $E_{n}$ is isomorphic to $l^{\infty}\left(S_{n}\right)$ with a bijection of norm $n$. Hence $\left\{a_{(k, n)}: k \in \mathrm{N}\right\}$ is limited in the strongest sense with $C=1 / n$.

Example 1. Let $\left\{a_{(k, n)}:(k, n) \in \mathrm{N} \times \mathrm{N}\right\}$ be the unit vectors of $c_{0}(\mathrm{~N} \times \mathbf{N})$ and let $E_{n}$ be the Banach space in Lemma 1 associated to $\left\{a_{(k, n)}: k \in \mathrm{N}\right\}$ but without the terms $\left\{f_{(\lambda, n)}: \lambda \in \Lambda_{n}\right\}$. Then $\left(a_{(k, n)}\right)_{k}$ is limited in $E_{n}$ according to Remark 3 and each $E_{n}^{\prime}$ (as in the construction preceeding Lemma 1) is naturally identified with the subspace $l^{\infty}(\mathrm{N} \times n)$ of $l^{\infty}(\mathrm{N} \times \mathrm{N})$ (because $\left.A_{n}=S_{n}=\mathrm{N} \times n\right)$ and $E_{n}$ is $l^{\infty}(\mathrm{N} \times n)$ equiped with the equivalent norm $\|z\|=\max \left[\|z\|_{l^{\infty}}, n\|z\|_{l^{\infty} / c_{0}}\right]$. Note that if $\varphi_{k}^{(n)} \in E_{n}^{\star}$ is the projection onto $\left[a_{(k, n)}\right]$ such that $\varphi_{k}^{(n)}\left(a_{(k, n)}\right)=1, \varphi_{k}^{(n)}\left(a_{(j, n)}\right)=0$ for $k \neq j$ and $\left\|\varphi_{k}^{(n)}\right\|=1$, then $\lim \sup _{k \rightarrow \infty}\left|\varphi_{k}^{(n)}(z)\right| \leq\|z\| / n$ for all $z \in E_{n}$.

Let $\Upsilon$ be the set of all sequences $v=\left(n, v_{n}\right)_{n} \subset \mathbf{N} \times \mathbf{N}$. Note that $l^{\infty}(v) \subset l^{\infty}(\mathrm{N} \times \mathbf{N})$ and that the unit vectors of $l^{\infty}(v)$ is limited in $l^{\infty}(v)$. Let $E$ be the sup-norm sum of the Banach spaces $\left\{E_{n}: n \in \mathrm{N}\right\}$ and the family $\left\{l^{\infty}(v): v \in \Upsilon\right\}$. Then $D=\left\{a_{(k, n)}:(k, n) \in \mathrm{N} \times \mathbf{N}\right\}$ is limited in $E$ (every sequence in $D$ has a subsequence in either some $E_{n}$ or in some $l^{\infty}(v)$ ). But $D$ is not limited in the strong sense since, for given $n$, the projections $\left\{\varphi_{k}^{(n)}: k \in \mathrm{N}\right\}$ above can be extended to $E$ by putting $\varphi_{k}^{(n)} / E_{j}=$ $0=\varphi_{k}^{(n)} / l^{\infty}\left(v \backslash\left\{n, v_{n}\right\}\right)$. Then $\left(\varphi_{k}^{(n)}\right)_{k} \subset E^{\star}$ is still norm bounded by 1 , $\sup _{z \in D}\left|\varphi_{k}^{(n)}(z)\right|=1$ but $\limsup _{k \rightarrow \infty}\left|\varphi_{k}(z)\right| \leq\|z\| / n$ for all $z \in E$. This holds for every $n$ and thus $D$ is not limited in the strong sense.

Note that every sequence in $D$ is limited even in the strongest sense (once again because every sequence in $D$ has a subsequence in either some $E_{n}$ or in some $l^{\infty}(v)$ ). Thus neither being limited in the strongest nor the strong sense is a sequential property.

The following definitions are however sequential.

Definition 2. A set $D \subset E$ is called uniformly limited if every sequence in $D$ has a subsequence uniformly limited in the strong sense.

Further $D$ is said to be strongly uniformly limited if every sequence in $D$ has a subsequence uniformly limited in the strongest sense. 
The class of uniformly limited sets is denoted $\mathscr{L}_{4}$ while $\mathscr{L}_{3}$ denotes the class of strongly uniformly limited sets.

The set $D$ in example 1 is strongly uniformly limited.

We shall now construct the example showing there is no way of defining the concept of uniform bounds such that it holds for all limited sets.

Let $S=\{(j, k) \in \mathrm{N} \times \mathrm{N}: j \leq k\}$ and $\left\{e_{(j, k)}\right\}$ be the unit vectors of $c_{0}(S)$. Put $a_{k}=\sum_{j=1}^{k} e_{(j, k)}$, then $\left\{a_{k}\right\}$ generates a Banach space isometric to $c_{0}$. Put $a_{(k, n)}=\sum_{j=1}^{n} e_{(j, k)}$ where $e_{(j, k)}=0$ if $j>k$ for convenience. Let, for fixed $n, F_{n}$ be generated by $\left\{a_{(k, n)} ; k \in \mathrm{N}\right\}$. Then $F_{n}$ is isometric to $c_{0}$ and we can take $E_{n}$ associated to $\left(a_{(k, n)}\right)_{k}$ as in Lemma 1.

Put $a_{k}^{(n)}=\sum_{j=n+1}^{k} e_{(j, k)}$. Let $\Upsilon$ be the set of all non-decreasing, unbounded sequences $v=\left(v_{k}\right)$ of positive integers, such that $v_{k} \leq k$ for all $k$. Let, for every $v \in \Upsilon, c_{0}(v)$ denote the Banach space, isometric to $c_{0}$, generated by $\left\{a_{k}^{\left(v_{k}\right)}\right\}_{k}$ and let $l^{\infty}(v) \subset l^{\infty}(S)$ denote the corresponding $l^{\infty}$-space generated by all sequences $\left(z_{k} a_{k}^{\left(v_{k}\right)}\right)_{k}$, where $\left(z_{k}\right)_{k} \in l^{\infty}$. Let further $F$ be generated by $\left\{a_{(k, n)}:(k, n) \in S\right\}$ and $\left\{a_{k}^{\left(v_{k}\right)}: v \in \Upsilon\right\}$. Then $F$ is a subspace of $c_{0}(S)$, actually the whole of $c_{0}(S)$.

We shall now add the spaces $\left\{E_{n}: n \in \mathbf{N}\right\}$ and $\left\{l^{\infty}(v): v \in \Upsilon\right\}$ in a different manner than in Example 1.

Recall that $E_{n}$, regarded as a vector space, is a subspace of $l^{\infty}\left(A_{n}\right)$, where $A_{n}=S_{n} \cup S_{n}^{\prime}$ and $l^{\infty}\left(S_{n}\right) \subset l^{\infty}(S)$. Thus $z \in E_{n}$ has a natural decomposition $z=z^{(1)}+z^{(2)}$ where $z^{(1)} \in l^{\infty}(S)$ and $z^{(2)} \in l^{\infty}\left(S_{n}^{\prime}\right)$. Furthermore \|\|$_{E_{n}}=$ $\max \left[\|\|_{l^{\infty}\left(A_{n}\right)}, n\|\|_{l^{\infty}\left(A_{n}\right) / F_{n}}\right]$. Let $E$ be the Banach space generated by the Banach spaces $\left\{E_{n}: n \in \mathbf{N}\right\}$ and $\left\{l^{\infty}(v): v \in \Upsilon\right\}$ in such a way that

$$
\begin{gathered}
\left\|\sum_{n=1}^{t} z_{n}+\sum_{l=1}^{r} z_{v_{l}}\right\|_{E}= \\
\max \left[\left\|\sum_{n=1}^{t} z_{n}^{(1)}+\sum_{l=1}^{r} z_{v_{l}}\right\|_{l^{\infty}(S)}, \sum_{n=1}^{t}\left\|z_{n}\right\|_{E_{n} / F_{n}}+\sum_{l=1}^{r}\left\|z_{v_{l}}\right\|_{l^{\infty}(S) / F}\right]
\end{gathered}
$$

where $z_{n} \in E_{n}$ and $z_{v_{l}} \in l^{\infty}\left(v_{l}\right)$

THEOREM 1. The sequence $\left(a_{k}\right)$ is limited in $E$. But for each $n$ there exists a sequence $\left(\varphi_{k}\right) \subset E^{\star}$ such that $\varphi_{k}\left(a_{k}\right)=1=\left\|\varphi_{k}\right\|$ and

$$
\sup _{\|z\|=1} \limsup _{k \rightarrow \infty}\left|\varphi_{k}(z)\right| \leq 1 / n
$$

Proof. Assume that $\left(a_{k}\right)$ is not limited. Then there exists a weak ${ }^{\star}$ null 
sequence $\left(\varphi_{k}\right)$ such that $\lim \sup \left|\varphi_{k}\left(a_{k}\right)\right|=1$. Put $C=\sup \left\|\varphi_{k}\right\|$. There does not exist $n>8 C$ such that $\limsup _{k \rightarrow \infty}\left|\varphi_{k}\left(a_{(k, n)}\right)\right| \geq 1 / 2$, since then, according to Lemma $1, \lim \sup _{k \rightarrow \infty}\left\|\varphi_{k}\right\| \geq n / 8>C$.

Thus we may take, for every $n>8 C, m_{n}>m_{n-1}$ such that $\left|\varphi_{k}\left(a_{(k, n)}\right)\right|<$ $1 / 2$ when $k \geq m_{n}$. Especially $\left|\varphi_{m_{n}}\left(a_{m_{n}}^{(n)}\right)\right|>1 / 2$ for every $n>8 C$. Thus $\lim \sup _{k \rightarrow \infty}\left|\varphi_{k}\left(a_{k}^{\left(\lambda_{k}\right)}\right)\right| \geq 1 / 2$, where $\lambda_{k}=n$ when $m_{n} \leq k<m_{n+1}$. Hence $\left(a_{k}^{\left(\lambda_{k}\right)}\right)_{k}$ is not limited in $E$, which is impossible since the unit vectors of $c_{0}$ is limited in $l^{\infty}$. Thus $\left(a_{k}\right)$ is limited in $E$.

To prove the estimate of the Theorem, fix $n$ and let $\varphi_{k} \in l^{\infty}(S)^{\star}$ be such that $P_{n, k}(z)=\varphi_{k}(z) e_{(n, k)}$ is the natural projection $l^{\infty}(S) \rightarrow\left[e_{(n, k)}\right]$. Thus $\varphi_{k}\left(e_{(n, k)}\right)=1=\left\|\varphi_{k}\right\|$. Obviously we can extend the definition of $\varphi_{k}$ by putting $\varphi_{k} / l^{\infty}\left(S_{j}^{\prime}\right)=0$ for every $j$. Thus we may assume that $\varphi_{k} \in E^{\star}$ and still $\left\|\varphi_{k}\right\|=1$. Further we have that $\varphi_{k} / E_{j}=0$ if $j<n$ and that $\left(\varphi_{k} / l^{\infty}(v)\right)$ is a weak $^{\star}$ null sequence in $l^{\infty}(v)^{\star}$ for every $v \in \Upsilon$, because $v_{k} \rightarrow \infty$ as $k \rightarrow \infty$.

Let $z=\sum_{j=1}^{t} z_{j}+\sum_{l=1}^{r} z_{v_{l}} \in E$, where $z_{j} \in E_{j}$ and $z_{v_{l}} \in l^{\infty}\left(v_{l}\right)$, be such that $\lim \sup _{k \rightarrow \infty}\left|\varphi_{k}(z)\right|>1 / n$. Since $\varphi_{k} / l^{\infty}\left(S_{j}^{\prime}\right)=0, \quad \varphi_{k} / E_{j}=0$ if $j<n$ and $\lim _{k \rightarrow \infty} \varphi_{k}\left(z_{v_{l}}\right)=0$ for every $l$ we get that

$$
\limsup _{k \rightarrow \infty}\left|\varphi_{k}\left(\sum_{j=1}^{t} z_{j}+\sum_{l=1}^{r} z_{v_{l}}\right)\right|=\limsup _{k \rightarrow \infty}\left|\varphi_{k}\left(\sum_{j=n}^{t} z_{j}^{(1)}\right)\right|>1 / n
$$

Then

$$
1<n\left\|\sum_{j=n}^{t} z_{j}^{(1)}\right\|_{l^{\infty}(S) / F} \leq n \sum_{j=n}^{t}\left\|z_{j}^{(1)}\right\|_{l^{\infty}(S) / F} \leq \sum_{j=n}^{t}\left\|z_{j}^{(1)}\right\|_{E_{n} / F} \leq\|z\|
$$

according to the definition of the norm in $E$. Hence $\lim \sup _{k \rightarrow \infty}\left|\varphi_{k}(z)\right| \leq 1 / n$ if $\|z\| \leq 1$ and the theorem is proved.

REMARK 4. Let $B$ be the unit ball of the $c_{0}$-space generated by $\left\{a_{k}: k \in \mathrm{N}\right\}$. Let $D \subset r B+K$, where $r>0$ and $K \subset E$ is compact, be not relatively compact. Then there exist a sequence $\left(b_{k}\right) \subset D$ and $d>0$ such that $\left\|b_{j}-b_{k}\right\| \geq d$ and $d \leq\left\|b_{k}\right\|$ for all $j \neq k$. The proof of Theorem 1 together with the basis properties of $c_{0}$ gives that there exists, for every given $n \in \mathrm{N}$, a sequence $\left(\varphi_{k}\right) \subset E^{\star}$, such that $\left\|\varphi_{k}\right\|=1,\left|\varphi_{k}\left(b_{k}\right)\right| \geq d$ but $\lim \sup _{k \rightarrow \infty}$ $\left|\varphi_{k}(z)\right| \leq\|z\| / n$ for every $z \in E$. Thus no set $D \subset r B+K$, besides the relatively compact ones, is limited with uniform bounds in any sense. Now $E$ given above contains limited sets which are limited even in the strongest sense, without being relatively compact, because $E$ has subspaces isomorphic to $l^{\infty}$. A somewhat more complicated example can be given so that only relatively compact sets are uniformly limited whatever uniform bound we use. 
We just briefly sketch how the construction of $E$ can be modified to give such a space.

Obviously we have to replace $l^{\infty}(v)$ by GP-spaces as in Lemma 1 . Let $\Upsilon$ and $P_{\infty}(\mathrm{N})$ be as before. Put $\Omega=\Upsilon \times P_{\infty}(\mathrm{N})$. To every $\omega=(v, M) \in \Omega$ we associate a sequence $\left(\omega_{n}\right)$, where $\omega_{n}=\left(\left[v_{k} / n\right]\right)_{k \in M}$ and [ ] denotes the integer part. Note that $\omega_{n}$, for every $n$, is a non-decreasing, unbounded sequence of positive integers. Let, for every $\omega_{n}, F_{\omega_{n}}$ denote the Banach space, isometric to $c_{0}$, generated by $\left\{a_{k}^{\left(\left[v_{k} / n\right]\right)}: k \in M\right\}$. We may assume that $\Omega$ is well-ordered and use transfinite induction. Assume that we have taken $E_{\kappa_{n}}$ for every $n$ and every $\kappa<\omega=(v, M)$. If there is $\kappa=\left(v^{(\kappa)}, M_{\kappa}\right)<\omega$ such that $E_{\kappa_{1}} \neq \emptyset$ and such that for some integer $n$ and some infinite set $U \subset M_{\kappa} \cap M$ we have that $\left[v_{k}^{(\kappa)} / n\right] \leq v_{k}$ when $k \in U$, we put $E_{\omega_{j}}=\emptyset$ for every $j$. Otherwise we take, for every $j, E_{\omega_{j}}$ associated to the set $\left\{a_{k}^{\left(\left[v_{k} / j\right]\right)}: k \in M\right\}$ as in Lemma 1 with $n$ replaced by $j$.

Let $E$ be the Banach space generated by the Banach spaces $\left\{E_{n}: n \in \mathrm{N}\right\}$ and $\left\{E_{\omega_{n}}: n \in \mathrm{N}\right.$ and $\left.\omega \in \Omega\right\}$ in such a way that

$$
\begin{gathered}
\left\|\sum_{n=1}^{t} z_{n}+\sum_{l=1}^{r} z_{\omega_{j_{l}}^{(l)}}\right\|_{E}= \\
\max \left[\left\|\sum_{n=1}^{t} z_{n}^{(1)}+\sum_{l=1}^{r} z_{\omega_{j_{l}}^{(l)}}^{(1)}\right\|_{l^{\infty}(S)}, \sum_{n=1}^{t}\left\|z_{n}\right\|_{E_{n} / F_{n}}+\sum_{l=1}^{r} \| z_{\left.\omega_{j_{l}}^{(l)} \|_{\sum_{\omega_{j_{l}}^{(l)} / F} \omega_{j_{l}}^{(l)}}\right]}\right]
\end{gathered}
$$

where $z_{n} \in E_{n}$ and $z_{\omega_{j_{l}}^{(l)}} \in E_{\omega_{j_{l}}^{(l)}}$ and $z_{\omega_{j_{l}}^{(l)}}^{(1)}$ is defined as $z_{n}^{(1)}$.

Essentially the same proof works to show that Theorem 1 also holds for this new Banach space $E$. It remains to show that if $D$ is limited in $E$, then $D \subset r B+K$ for some $r>0$ and some compact set $K \subset E$. The main difficulty is to show that a set of type $\left\{a_{k}^{\left(v_{k}\right)}: k \in M\right\}$, where $v \in \Upsilon$, is limited. Take $\omega=(v, M)$. If $E_{\omega_{1}} \neq \emptyset$ there exists a weak ${ }^{\star}$ null sequence $\left(\varphi_{k}\right)_{k \in M} \subset E_{\omega_{1}}^{\star}$ such that $\varphi_{k}\left(a_{k}^{\left(v_{k}\right)}\right)=1$ when $k \in M$. We give, for every $k \in M, \varphi_{k}$ the additional properties that $\varphi_{k}\left(e_{(i, k)}\right)=\varphi_{k}\left(e_{(j, k)}\right)$ if $i>j>v_{k}, \varphi_{k}\left(e_{\left(v_{k}, k\right)}\right)=-1$ and $\varphi_{k}\left(e_{(j, p)}\right)=0$ for all other $(j, p) \in S$. Observing that if both $E_{\omega_{1}}$ and $E_{\kappa_{1}}$ are nonempty and $V=M \cap M_{\kappa}$ then either $\lim _{\{k \in V, k \rightarrow \infty\}} v_{k} / v_{k}^{(\kappa)}=0$ or $\lim _{\{k \in V, k \rightarrow \infty\}} v_{k}^{(\kappa)} / v_{k}=0$, it is easy to verify that $\left(\varphi_{k}\right)_{k \in M}$ is a weak ${ }^{\star}$ null sequence in every $E_{\kappa_{n}}^{\star}$ and every $E_{n}^{\star}$ and hence in $E^{\star}$. If $E_{\omega_{1}}=\emptyset$ (and hence all $E_{\omega_{j}}=\emptyset$ ) we take $E_{\kappa_{n}}$ (at least one exists) that forces $E_{\omega_{1}}$ to be the empty set and $U \subset M_{\kappa} \cap M$ as above. Then take a weak ${ }^{\star}$ null sequence $\left(\varphi_{k}\right)_{k \in U} \subset E_{\kappa_{n}}^{\star}$ such that $\varphi_{k}\left(a_{k}^{\left(v_{k}\right)}\right)=1, \varphi_{k}\left(e_{(i, k)}\right)=\varphi_{k}\left(e_{(j, k)}\right)$ when $i>j>v_{k}, \varphi_{k}\left(e_{\left(v_{k}, k\right)}\right)=-1$ and proceed as above.

The following Lemma will be used in the next section. 
Lemma 2. Let $D$ be a non-uniformly limited set in the Banach space E. Then there exist a sequence $\left(a_{k}\right) \subset D$ and a constant $K>0$ such that, for every $n$, there exists $\left(\varphi_{k}^{(n)}\right) \subset E^{\star}$ norm bounded by $K$ with $\varphi_{k}^{(n)}\left(a_{k}\right)=1$, for $\forall n$ and $k$, but $\sup _{\|z\|=1} \lim \sup _{k \rightarrow \infty}\left|\varphi_{k}^{(n)}(z)\right| \leq 1 / n$.

Proof. If $D$ is non-uniformly limited there exist a sequence $\left(a_{k}\right) \subset D$ and a constant $K$ such that, for each integer $n$, there exists $\left(\varphi_{k}^{(n)}\right) \subset E^{\star}$ with $\left\|\varphi_{k}^{(n)}\right\|<K, \quad \lim \sup _{k \rightarrow \infty}\left|\varphi_{k}^{(n)}\left(a_{k}\right)\right|=1$ but $\quad \lim \sup _{k \rightarrow \infty}\left|\varphi_{k}^{(n)}(z)\right|<\|z\| / n$ if $z \neq 0$. A subsequence of a subsequence passing to the diagonal argument shows that we may assume that this holds for all subsequences of $\left(a_{k}\right)$. But then once again subsequences out of subsequences and passing to the diagonal give us a sequence, also denoted $\left(a_{k}\right)$, such that for every given $n$ there exist $\left(\varphi_{k}^{(n)}\right)$, norm bounded by $K$, such that $\lim _{k \rightarrow \infty}\left|\varphi_{k}^{(n)}\left(a_{k}\right)\right|=1$ but $\limsup _{k \rightarrow \infty}\left|\varphi_{k}^{(n)}(z)\right|<\|z\| / n$ for every $z \in E, z \neq 0$. To get the sequence in the Lemma we just have to notice that we are free to change finitely many $\varphi_{k}^{(n)}$ for every fixed $n$.

REMARK 5. If $D$ is not even limited we may take $\left(\varphi_{k}^{(n)}\right)$ independent of $n$. Also sets which are not strongly uniformly limited have a corresponding description (just omit $K$ and the proof will be essentially the same). Obviously Lemma 2 gives both a necessary and sufficient condition for a set to be not uniformly limited.

\section{Bounding sets}

First we reformulate the definition of being bounding in a way similar to the different classes of limited sets.

Lemma 3. A set D in a Banach space $E$ is bounding if and only if there exist, for every given sequence $\left(Q_{k}\right)_{k}$ of $j_{k}$-homogeneous polynomials on $E$ such that $\lim \sup _{k \rightarrow \infty} \sup _{z \in D}\left|Q_{k}(z)\right|^{1 / j_{k}}>0$, a constant $C>0, z \in E$ and a sequence $\left(z_{k}\right)_{k} \subset$ E such that $\lim _{k \rightarrow \infty} z_{k}=0$ and $\lim \sup _{k \rightarrow \infty} C^{j_{k}}\left|Q_{k}\left(z+z_{k}\right)\right|>0$.

The proof is simple.

Combining propositions from [1] and [8] we get:

Proposition 1.

1. The absolutely closed convex hull of a limited set is limited.

2. Subsets of a limited set are limited.

3. The set $A_{1}-A_{2}=\left\{a_{1}-a_{2}: a_{1} \in A_{1}, a_{2} \in A_{2}\right\}$ is limited if $A_{1}$ and $A_{2}$ are limited and so is $t A_{1}$ for every $t \in \mathrm{C}$.

4. Limited sets are conditionally weakly compact. 
5. Limited subsets of spaces containing no copy of $l_{1}$ are relatively weakly compact.

6. If there is, for each $\epsilon>0$, a limited set $D_{\epsilon}$ such that that $D \subset D_{\epsilon}+\epsilon B_{E}$, then $D$ is limited in $E$.

Since $\mathscr{L}$ is the largest class, 4) and 5) hold for all classes considered here and evidently also 2 ). It is immediate that the classes $\mathscr{L}_{1}$ and $\mathscr{L}_{2}$ are closed under taking convex hulls and by Lemma 2 it is easily seen that also $\mathscr{L}_{3}$ and $\mathscr{L}_{4}$ have the hull-property. Property 3 ) is merely a special case of 1 ) and holds for bounding sets, though not as obvious as in the limited case. Let $A$ and $B$ be bounding sets. If $A+B$ is not bounding there exist a holomorphic function $f(z)=\sum_{k=1}^{\infty} P_{k}(z)$, where $P_{k}: E \rightarrow \mathrm{C}$ is a $j_{k}$-homogeneous polynomial, and sequences $\left(a_{k}\right)_{k} \subset A$ and $\left(b_{k}\right)_{k} \subset B$ such that $P_{k}\left(a_{k}+b_{k}\right)=$ $j_{k}+1$ for every $k$. Write $P_{k}\left(a_{k}+b_{k}\right)=\sum_{s=0}^{j_{k}} Q_{s, k}\left(a_{k}, b_{k}\right)$ where $Q_{s, k}\left(a_{k}, b_{k}\right)$ is a $s$-homogeneous polynomial in $a_{k}$ and a $\left(j_{k}-s\right)$-homogeneous polynomial in $b_{k}$. Since $P_{k}\left(a_{k}+b_{k}\right)=j_{k}+1$ there exists, for every $k$, at least one $s_{k}$ such that $\left|Q_{s_{k}, k}\left(a_{k}, b_{k}\right)\right| \geq 1$. Since $\left(a_{k}\right)$ is bounding there exist, according to Lemma $3, \quad C>0, a \in E$ and $\left(x_{k}\right)_{k} \subset E$ such that $\lim _{k \rightarrow \infty} x_{k}=0$ and $\lim \sup _{k \rightarrow \infty} C^{s_{k}}\left|Q_{s_{k}, k}\left(a+x_{k}, b_{k}\right)\right|>0$. Since $\left(b_{k}\right)_{k}$ is bounding the same argument gives $b \in E$ and $\left(y_{k}\right)_{k} \subset E$ such that $\lim _{k \rightarrow \infty} y_{k}=0$ and $\lim \sup _{k \rightarrow \infty}\left|Q_{s_{k}, k}\left(a+x_{k}, b+y_{k}\right)\right|^{1 / j_{k}}>0$. But then a Cauchy-estimate shows that $f(z)$ is not holomorphic and we get a contradiction which proves 3 ) for bounding sets. (A similar argument shows that 3 ) holds for all classes of bounding sets considered here.) On the other hand bounding sets does not in general have a bounding convex hull as the following example shows.

ExAmple 2. Let, for each $n \in \mathrm{N},\left\{e_{(k, n)}: k \in \mathrm{N}, k \geq n\right\}$ be an isometric copy of the unit vectors of $c_{0}$ and, for each fixed $k \in \mathrm{N},\left\|\sum_{n=1}^{k} t_{n} e_{(k, n)}\right\|=$ $\sum\left|t_{n}\right|$. Put $S=\{(k, n) \in \mathrm{N} \times \mathrm{N}: k \geq n\}$. Let

$$
G=\left\{z: z=\sum_{(k, n) \in S} z_{(k, n)} e_{(k, n)}\right\}
$$

with $\|z\|=\sup _{k} \sum_{n=1}^{k}\left|z_{(k, n)}\right|$. Denote by $H_{n}$ the $l^{\infty}$-space generated by $\left\{e_{(k, n)}: k \in \mathrm{N}, k \geq n\right\}$ and by $c_{0, n}$ the corresponding $c_{0}$-space. Let $\Upsilon$ be the set of all sequences $v=\left(v_{n}, n\right)_{n} \subset S$ such that $v_{n}<v_{n+1}$ for all $n \in \mathrm{N}$. Let $l^{\infty}(v)$ denote the $l^{\infty}$-space generated by $\left\{e_{\left(v_{n}, n\right)}: n \in \mathrm{N}\right\}$ and note that $l^{\infty}(v) \subset G$. Let further $c_{0}(v)$ be the corresponding $c_{0}$-space. Let finally $E \subset G$ be the Banach space generated by $\left\{H_{n}: n \in \mathrm{N}\right\}$ and the family $\left\{l^{\infty}(v): v \in \Upsilon\right\}$, i.e., the closure in $G$ of the set $\left\{z=\sum_{n=1}^{r} z_{n}+\sum_{i=1}^{s} z_{v_{i}}: z_{n} \in H_{n}, z_{v_{i}} \in l^{\infty}\left(v_{i}\right)\right\}$. Then $D=\left\{e_{(k, n)}:(k, n) \in S\right\}$ is limited in the strongest sense in $E$ since the unit vectors of $c_{0}$ is limited in the strongest sense in $l^{\infty}$. Hence the closed 
absolute convex hull $\widehat{D}$ of $D$ also is limited in the strongest sense. Furthermore $D$ is bounding, even strongly bounding, since the unit vectors of $c_{0}$ is (strongly) bounding in $l^{\infty}$. (For the notion of strongly bounding see definition 3 and remark 6 below.) We shall prove that $\widehat{D}$ is not bounding, more precisely that $\left\{a_{k}: k \in \mathrm{N}\right\}$, where $a_{k}=\frac{1}{k} \sum_{n=1}^{k} e_{(k, n)}$, is not bounding.

Put $f(z)=\sum_{k=1}^{\infty} P_{k}(z)=\sum_{k=1}^{\infty} k^{k+1} \prod_{n=1}^{k} \varphi_{k, n}(z)$, where $\varphi_{k, n} \in E^{\star}$, for every $(k, n)$, is the natural norm-one projection of $E$ onto the span $\left[e_{k, n}\right]$. Then $f\left(a_{k}\right)=k \rightarrow \infty$ as $k \rightarrow \infty$ because $k^{k+1} \prod_{n=1}^{k} \varphi_{k, n}\left(a_{k}\right)=k$. Let $z=\sum_{n=1}^{r} z_{n}+$ $\sum_{i=1}^{s} z_{v_{i}}=\sum_{(k, n) \in S} z_{(k, n)} e_{(k, n)} \in E$ and take $\epsilon>0$. There exist an integer $m>0$ and, for every $k \in \mathrm{N}$, a set $M_{k} \subset\{1,2, k\}$ with at most $m$ elements such that $\sum_{n \in\{1, k\} / M_{k}}\left|z_{(k, n)}\right|<\epsilon$ for every $k$. Then $\sup _{\|x\|<\epsilon}\left|P_{k}(z+x)\right|<$ $k(k(\|z\|+\epsilon))^{m}(2 \epsilon)^{k-m}\left(\frac{k}{k-m}\right)^{k-m}<(3 \epsilon)^{k}$, if $k$ is big enough, according to the relation between the geometrical and the arithmetical mean. Thus $f$ is an entire function and $\left\{a_{k}: k \in \mathrm{N}\right\}$ is not bounding.

Property 6) is an analogy of Grothendieck's characterization of relatively weakly compact set as pointed out in [8] and can, by Lemma 2, easily be generalised to the classes $\mathscr{L}_{2}$ and $\mathscr{L}_{4}$ i.e., if $D$ can be $\epsilon$-incapsulated by uniformly limited sets (in the strong sense) then $D$ itself is a uniformly limited set (in the strong sense). Neither bounding sets nor strongly uniformly limited sets have in general this property.

Example 3. Let $\left\{e_{(k, n)}:(k, n) \in \mathrm{N} \times \mathrm{N}=S\right\}$ be vectors such that, for each fixed $n \in \mathrm{N}$, the set $\left\{e_{(k, n)}: k \in \mathrm{N}\right\}$ is isometric to the unit vectors of $c_{0}$ and such that, for each $k \in \mathrm{N},\left\{e_{(k, n)}: n \in \mathrm{N}\right\}$ is isometric to the unit vectors of $l_{1}$. Let $H_{n}$ be the $l^{\infty}$-space generated by $\left\{e_{(k, n)}: k \in \mathrm{N}\right\}$ and let $G$ be the Banach space generated by $\left\{H_{n}: n \in \mathrm{N}\right\}$ equiped with the norm $\|z\|_{G}=\left\|\sum_{n=1}^{j} z_{n}\right\|=$ $\sup _{k \in \mathrm{N}} \sum_{n=1}^{j}\left|z_{(k, n)}\right|$ where $z_{n}=\left(z_{(k, n)}\right)_{k} \in H_{n}$. Put $a_{(k, n)}=e_{(k, 1)}+c_{n} e_{(k, n+1)}$, where $c_{n} \in \mathrm{R}$ will be determined below. Then $\left\{a_{(k, n)}: k \in \mathrm{N}\right\}$ generates, for every $n \in \mathrm{N}$, a Banach space $F_{n}$ isometric to $c_{0}$ and $\lim _{n \rightarrow \infty} a_{(k, n)}=e_{k, 1}$ if $\lim _{n \rightarrow \infty} c_{n}=0$. Let $F_{n}^{\infty}$ be the $l^{\infty}$-space generated by to $\left(a_{(k, n)}\right)_{k}$. Let $F$ be the Banach space generated by $\left\{a_{(k, n)}:(k, n) \in S\right\}$. Give $F_{n}^{\infty}$ the equivalent norm $\|z\|=\max \left[\|z\|_{F_{n}^{\infty}}, d_{n}\|z\|_{F_{n}^{\infty} / F_{n}}\right.$, where $d_{n} \in \mathrm{R}, d_{n}>1$ will be determined later, and let $E_{n}$ be $F_{n}^{\infty}$ equiped with this new norm. Note that $\|z\|=\|z\|_{F_{n}^{\infty}}$ if $z \in F_{n}$. Note also that if $\left(\varphi_{k}\right) \subset E^{\star}$ is such that $\limsup _{k \rightarrow \infty}\left|\varphi_{k}\left(a_{(k, n)}\right)\right|^{n}=1$ then $\lim \sup _{k \rightarrow \infty}\left|\varphi_{k}(z)\right| \geq \frac{1}{d_{n}}\|z\|$ for some $z \in E_{n}$ (because the unit vectors are limited in the strongest sense in $\left.l^{\infty}\right)$. Thus $\left(a_{(k, n)}\right)_{k}$ is limited in the strongest sense in $E_{n}$ and is also bounding because $E_{n}$ is isomorphic to $F_{n}^{\infty}$ and the unit vectors are bounding in $l^{\infty}$. Let finally $E$ be the Banach space generated by $\left\{E_{n} ; n \in \mathrm{N}\right\}$ in such a way that if $z=\sum_{i=1}^{j} z_{i} \in E$, where $z_{i} \in E_{i}$, then $\|z\|_{E}=\max \left[\|z\|_{G}, \sum_{i=1}^{j}\left\|z_{i}\right\|_{E_{n} / F_{n}}\right]$. Note that $F$ is isometric to a subspace of $E$ that we identify with $F$. Put now $c_{n}=1 / n$ and $d_{n}=n^{n}$. Then $\left(e_{(k, 1)}\right)_{k}$ is uni- 
formly limited in $E$ since uniformly limited sets have the G-property according to above and obviously $\left(a_{(k, n)}\right)_{k}$ is bounding also in $E$ for every $n$.

Define $\varphi_{k, n} \in E^{\star}$ by $\varphi_{k, n} / E_{s}=0$ when $s \leq n$, and $\varphi_{k, n} / E_{s}$ by the norm-one projection onto $\left[a_{(k, n)}\right]$ if $s>n$. Then it is readily verified that $\varphi_{k, n}\left(e_{(k, 1)}\right)=1$ and that $\left\|\varphi_{k, n}\right\|=n$ because $\mid \varphi_{k, n}\left(e_{(k, s+1)} \mid=s\right.$ if $s \leq n$ and $\mid \varphi_{k, n}\left(e_{(k, s+1)} \mid=0\right.$ if $s>n$. Furthermore $\lim \sup _{k \rightarrow \infty}\left|\varphi_{k, n}(z)\right| \leq\|z\| / s^{s}$ if $z \in E_{s}$, where $s>n$, since $\left|\varphi_{k, n}(z)\right| \leq \frac{1}{s^{s}}$ when $s>n, z \in E_{s}$ and $\|z\|=\|z\|_{E_{s} / F_{s}}=1$. Thus the $l_{1}$ sum gives that $\lim \sup _{k \rightarrow \infty}\left|\varphi_{k, n}(z)\right| \leq\|z\| / n^{n}$ for all $z \in E$. Since this is true for every $n \in \mathrm{N}$ we conclude that $\left\{e_{(k, 1)}: k \in \mathrm{N}\right\}$ is not limited in the strongest sense and hence neither strongly uniformly limited since $\lim \sup _{k \rightarrow \infty} \sup _{z \in D}$ $\left|\varphi_{k, n}(z)\right|=1$ for every subsequence $D$ of $\left(e_{(k, 1)}\right)$.

Put $f(z)=\sum_{k=1}^{\infty} k \prod_{n=1}^{k} \varphi_{k, n}(z)$. Then $f$ is an entire function because, for each $k \in \mathrm{N},\left\{e_{(k, n)}: n \in \mathrm{N}\right\}$ is isometric to the unit vectors of $l_{1}$ (see the end of example 2) and because $\left|\varphi_{k, n}(z)\right| \leq\|z\| / n^{n}$ for every $k$ and $n$ if $\|z\|=\|z\|_{E / F}$. It is obvious that $f\left(e_{(k, 1)}\right)=k \rightarrow \infty$ as $k \rightarrow \infty$. Thus $\left\{e_{(k, 1)}: k \in \mathrm{N}\right\}$ is not bounding.

The construction above can be modified to give a bounding set which is not strongly uniformly limited.

Example 4. Let $\left\{e_{(k, n)}:(k, n) \in \mathrm{N} \times \mathrm{N}=S\right\}$ be the unit vectors of $c_{0}(S)$. Put $a_{(k, n)}=e_{(k, 1)}+n^{-2^{2^{n}}} e_{(k, n+1)}$. As in the preceding example $\left\{a_{(k, n)}: k \in \mathrm{N}\right\}$ generates, for every $n \in \mathrm{N}$, a Banach space $F_{n}$ isometric to $c_{0}$ and $\lim _{n \rightarrow \infty} a_{(k, n)}=e_{(k, 1)}$. Let $H_{n}$ be the $l^{\infty}$-space belonging to $\left(a_{(k, n)}\right)_{k}$ and define a equivalent norm by $\|z\|=\max \left[\|z\|_{H_{n}}, n\|z\|_{H_{n} / F_{n}}\right]$, and let $E_{n}$ denote $H_{n}$ equiped with this new norm. Let finally $E$ be the Banach space generated by $\left\{E_{n} ; n \in \mathrm{N}\right\}$ in such a way that if $z=\sum_{i=1}^{j} z_{i} \in E$, where $z_{i} \in E_{i}$, then $\|z\|=\max \left[\|z\|_{l^{\infty}(S)}, \sum_{i=1}^{j}\left\|z_{i}\right\|_{E_{n} / F_{n}}\right]$. Note that $c_{0}(S)$ is a subspace of $E$. Then it follows exactly as in Example 3 that $\left(e_{(k, 1)}\right)_{k}$ is not strongly uniformly limited in $E$.

Assume that $\left(e_{(k, 1)}\right)_{k}$ is not bounding for holomorphic functions. Then there exist a holomorphic function $f(z)=\sum_{k=1}^{\infty} P_{j_{k}}(z)$, where $P_{j_{k}}$ is a $j_{k^{-}}$ homogeneous polynomial on $E$, such that $\limsup _{k \rightarrow \infty}\left|P_{j_{k}}\left(e_{(k, 1)}\right)\right|>1$ and a constant $C>0$ such that $\left\|P_{j_{k}}\right\|<C^{j_{k}}$ for every k. Take $m \in \mathrm{N}$ so big that $6 C / m<1 / 4$ and $m 4^{-m}<2^{-m}$. Let $D_{k}$ be the compact set $D_{k}=$ $\left\{\sum_{n=m}^{\infty} z_{n} a_{(k, n)} / 2^{n-m}: z_{n} \in \mathrm{C}\right.$ and $\left.\left|z_{n}\right| \leq 5 / 4 \forall n\right\}$. Let $B_{k}$ be the unit ball of the Banach space generated by $\left\{e_{(k, n)}: n \in \mathrm{N}\right\}$ and let $\left(\epsilon_{k}\right)$ be a sequence such that $\lim _{k \rightarrow \infty} \epsilon_{k}=0$.

We claim that

$$
\lim _{k \rightarrow \infty} \sup _{z \in D_{k}+\epsilon_{k} B_{k}}\left|P_{j_{k}}(z)\right|^{1 / j_{k}}=0
$$


Assume that this is not the case. Then there exist, since each $D_{k}$ is compact and actually translates of each other, sequences $\left(a_{k}\right)$ and $\left(x_{k}\right)$ in $E$ such that $\lim _{k \rightarrow \infty} x_{k}=0$ and such that, for every $k, a_{k} \in D_{k}, a_{k}$ can be written $a_{k}=\sum_{n=m}^{\infty} z_{n} a_{(k, n)} / 2^{n-m}$, where $z_{n} \in \mathrm{C}$ is independent of $k,\left|z_{n}\right| \leq 5 / 4$ and $\lim \sup _{k \rightarrow \infty}\left|P_{j_{k}}\left(a_{k}+x_{k}\right)\right|^{1 / j_{k}}>0$. Thus $\left(a_{k}\right)$ is not bounding according to Lemma 3. But $\left\{a_{k}\right\}$ is isomorphic to the unit vectors of $c_{0}$ and the $l^{\infty}$-space of all bounded sequences of $\left(a_{k}\right)_{k}$ is a subspace of $E$ because $E$ is the $l_{1}$-direct sum $\sum_{n=1}^{\infty} \oplus E_{n}$ and because $\sum_{n=m}^{\infty} n / 2^{n-m}$ is convergent. Hence $\left(a_{k}\right)$ is a bounding set and we get a contradiction.

We shall now show that 2$)$ is false and get a contradiction. Let $j_{k}>2^{2^{m}}$ be such that $\left|P_{j_{k}}\left(e_{(k, 1)}\right)\right|>1$. Put $Q(u)=P_{j_{k}}\left(e_{(k, 1)}-u e_{(k, m+1)} m^{-2^{2^{m}}}\right)=\sum_{p=0}^{j_{k}} d_{p} u^{p}$ and $Q^{(1)}(u)=\sum_{p=0}^{j_{k} / 2^{2^{m}}} d_{p} u^{p}$. Then $|Q(0)|=\left|Q^{(1)}(0)\right|>1$. Apply Lemma 4 below and we get that

$$
\sup _{|z| \leq \frac{1}{4}}\left|Q^{(1)}(1+z)\right| \geq \frac{2}{2^{j_{k} / 2^{m}}}
$$

Since $\sup _{|u| \leq m^{2^{2 m}}}|Q(u)| \leq C^{j_{k}}$ we have that $\left|d_{p}\right| m^{2^{2^{m}} p} \leq C^{j_{k}}$ which gives, for $p>j_{k} / 2^{2^{m}}$, that $\left|d_{p}\right|<(C / m)^{j_{k}}<4^{-j_{k}}$ and hence that $j_{k}\left|d_{p}\right|<2^{-j_{k}}$ because $j_{k}>m$. Thus

$$
\begin{gathered}
\sup _{|z| \leq \frac{1}{4}}|Q(1+z)|=\sup _{|z| \leq \frac{1}{4}}\left|P_{j_{k}}\left(e_{(k, 1)}-(1+z) e_{(k, m+1)} m^{-2^{2^{m}}}\right)\right|= \\
\sup _{|z| \leq \frac{1}{4}}\left|P_{j_{k}}\left((1+z) a_{(k, m)}-z e_{(k, 1)}\right)\right| \geq \frac{1}{2^{j_{k} / 2^{m}}}
\end{gathered}
$$

Thus there exist $z_{m} \in \mathrm{C}$ with $\left|z_{m}\right| \leq 5 / 4$ and $x_{m} \in \mathrm{C}$, with $\left|x_{m}\right| \leq 1 / 4$ such that $\left|P_{j_{k}}\left(z_{m} a_{(k, m)}+x_{m} e_{(k, 1)}\right)\right| \geq \frac{1}{2^{2 k} /^{2 m}}$. Assume now that we, for $n \geq m$ such that $2^{2^{n}}<j_{k}$, have taken $v_{(k, n)}=\sum_{s=m}^{n} z_{s} a_{(k, s)}$, where $z_{s} \in \mathrm{C}$ and $\left|z_{s}\right| \leq \frac{5}{4} 2^{s-m}$, and $x_{n} \in \mathrm{C}$, where $\left|x_{n}\right| \leq 1 / 2^{n-m}$, such that $\left|P_{j_{k}}\left(v_{(k, n)}+x_{n} e_{(k, 1)}\right)\right| \geq$ $1 / 2^{j_{k}} \sum_{s=m}^{n} 2^{-s}$. This is true for $n=m$ according to the above.

Put

$$
Q(u)=2^{j_{k} \sum_{s=m}^{n} 2^{-s}} P_{j_{k}}\left(v_{(k, n)}+x_{n} e_{(k, 1)}-u e_{(k, n+2)}(n+1)^{-2^{2^{n+1}}}\right)=\sum_{p=0}^{j_{k}} d_{p} u^{p}
$$

and $Q^{(1)}(u)=\sum_{p=0}^{j_{k} / 2^{2^{n+1}}} d_{p} u^{p}$. Then $|Q(0)|=\left|Q^{(1)}(0)\right| \geq 1$. If $2^{2^{n+1}}<j_{k}$ we get that 
UNIFORM BOUNDS FOR LIMITED SETS AND APPLICATIONS...

$$
\sup _{|u| \leq(n+1)^{2^{2 n+1}}}|Q(u)|=
$$

$$
2^{j_{k} \sum_{s=m}^{-s}} \sup _{|u| \leq(n+1)^{2^{2 n+1}}}\left|P_{j_{k}}\left(v_{(k, n)}+x_{n} e_{(k, 1)}-u e_{(k, n+2)}(n+1)^{-2^{2 n+1}}\right)\right| \leq(6 C)^{j_{k}}
$$

because $\sup _{|u| \leq(n+1)^{2^{2 n+1}}}\left\|v_{(k, n)}+x_{n} e_{(k, 1)}-u e_{(k, n+2)}(n+1)^{-2^{2^{n+1}}}\right\| \leq 3$. For the coefficients $d_{p}$ we this time get the estimate $\left|d_{p}\right|(n+1)^{2^{2^{n+1}} p} \leq(6 C)^{j_{k}}$ which gives, for $p>j_{k} / 2^{2^{n+1}}$, once again that $\left|d_{p}\right|<4^{-j_{k}}$ and hence that $j_{k}\left|d_{p}\right|<2^{-j_{k}}$ as before. Thus we can proceed as above, apply Lemma 4 and take $v_{(k, n+1)}=\sum_{s=m}^{n+1} z_{s} a_{(k, s)}$, where $z_{s} \in \mathrm{C}$ and $\left|z_{s}\right| \leq 2^{s-m} 5 / 4$, and $x_{n+1} \in \mathrm{C}$, where $\left|x_{n+1}\right| \leq 1 / 2^{n+1-m}$, such that $\left|P_{j_{k}}\left(v_{(k, n+1)}+x_{n+1} e_{(k, 1)}\right)\right| \geq 1 / 2^{j_{k} \sum_{s=m}^{n+1} 2^{-s}}$. Let $l_{k}$ be the biggest integer such that $2^{2^{l_{k}}}<j_{k}$. Then $v_{\left(k, l_{k}\right)}$ and $x_{l_{k}}$ exist as above. Note that there exist infinitely many $j_{k}$ such that $\left|P_{j_{k}}\left(e_{(k, 1)}\right)\right|>1$, that $l_{k} \rightarrow \infty$ as $j_{k} \rightarrow \infty$, that each $v_{\left(k, l_{k}\right)} \in D_{k}$, that $\epsilon_{k}=\left\|x_{l_{k}} e_{(k, 1)}\right\| \rightarrow 0$ as $k \rightarrow \infty$ and finally that $\left(1 / 2^{j_{k}} \sum_{s=m}^{\infty} 2^{-s}\right)^{1 / j_{k}}=1 / 2^{\sum_{s=m}^{\infty} 2^{-s}} \geq 1 / 2$. But this contradicts 2). Thus $\left\{e_{(k, 1)}\right\}$ is a bounding set in $E$.

Lemma 4. Let $n$ and $j$ be integers such that $n>10$ and $j>2^{2^{n}}$. Let $Q(z)$ be a polynomial in one complex variable of degree at most $j / 2^{2^{n}}$ such that $|Q(0)| \geq 1 / 2$. Then $\sup _{|z| \leq \frac{1}{4}}|Q(1+z)| \geq \frac{1}{2^{1 / 2^{n}}}$.

Proof. Let $w \in \mathrm{C}$ be such that $|w|=1$ and $|Q(w)|=\sup _{|z|=1}|Q(z)|=m$. Note that $m \geq\left|d_{p}\right|$ where $Q(z)=\sum_{p=0}^{j / 2^{2^{n}}} d_{p} z^{p}$. Assume that

$$
\sup _{|z| \leq \frac{1}{4}}|Q(1+z)| \leq \frac{1}{2^{j / 2^{n}}}
$$

and put $r(z)=\frac{2^{2^{n}}}{j} \ln |Q(w+z)|$. Since $|1-w| \leq 2$ we have, for $\theta \in\left[-\frac{1}{10}, \frac{1}{10}\right]$, that $s(\theta)=r\left(e^{i \theta}(1-w)\right)<\frac{2^{2^{n}}}{j} \frac{j}{2^{n}} \ln 1 / 2=-2^{2^{n}-n} \ln 2$. Note that

$$
\sup _{\theta}|s(\theta)| \leq \frac{2^{2^{n}}}{j} \ln m j 2^{j / 2^{2^{n}}}=\ln 2+\frac{2^{2^{n}}}{j} \ln m j
$$

because $\left|d_{p}\right| \leq m$ and there at most $j / 2^{2^{n}}+1<j$ terms in $Q$. Furthermore $r(z)$ is subharmonic. Thus

$$
\frac{2^{2^{n}}}{j} \ln |Q(w)|=\frac{2^{2^{n}}}{j} \ln m \leq \frac{1}{2 \pi} \int_{0}^{2 \pi} s(\theta) d \theta \leq \ln 2+\frac{2^{2^{n}}}{j} \ln m j-\frac{1}{10 \pi} 2^{2^{n}-n} \ln 2
$$

and hence 


$$
\ln 2+\frac{2^{2^{n}}}{j} \ln j-\frac{1}{10 \pi} 2^{2^{n}-n} \ln 2>0
$$

which implies, since $j>2^{2^{n}}$ and since $\frac{\ln x}{x}$ is a decreasing function for $x>e$, that

$$
\ln 2+\frac{2^{2^{n}}}{2^{2^{n}}} 2^{n} \ln 2-\frac{1}{10 \pi} 2^{2^{n}-n} \ln 2>0
$$

But this is impossible since $n>10$. Thus we got a contradiction and the Lemma is proved.

\section{Uniform limitedness and bounding sets}

Though limited sets in general are not uniformly limited we have the following:

THEOREM 2. Bounding sets in Banach spaces are uniformly limited

Proof. Let $D \subset E$ be a non-uniformly limited set. Take, according to Lemma 2, a sequence $\left(a_{k}\right)$ and a constant $C$ such that there exists, for every $n$, a sequence $\left(\varphi_{k, n}\right)_{k} \subset E^{\star}$, norm bounded by $C$, with $\varphi_{k, n}\left(a_{k}\right)=1$ for all $k \in \mathrm{N}$, but $\lim \sup _{k \rightarrow \infty}\left|\varphi_{k, n}(z)\right| \leq\|z\| /(4 C(n+1))^{2^{n}}$ for every $z \in E$.

Put $\quad P_{k}(z)=\prod_{r=2}^{k}\left(\varphi_{k, r}(z)\right)^{2^{k-r}}$. Let $z \in E$ be given and take $m>\max (\|z\|, C, 2)$. Take $k>m$ so big that

$$
\left|\varphi_{k, m}(z)\right| \leq \frac{2\|z\|}{(4 C(m+1))^{2^{m}}}
$$

Then

$$
\begin{aligned}
\sup _{\|y\| \leq(4 C(m+1))^{-2^{m}}}\left|\varphi_{k, m}(z+y)\right| & <\frac{2\|z\|}{(4 C(m+1))^{2^{m}}}+\frac{C}{(4 C(m+1))^{2^{m}}} \\
& <\frac{3 m}{(4 C(m+1))^{2^{m}}}
\end{aligned}
$$

Further

$$
\sup _{\|y\| \leq(4 C(m+1))^{-2^{m}}}\left|\varphi_{k, r}(z+y)\right|<C(m+1)
$$

for all $r$. Note that, the degree of $P_{k}, \sum_{r=2}^{k} 2^{k-r}<2^{k} / 4$. Thus (3) and (4) give the estimate 


$$
\begin{aligned}
& \sup _{\|y\| \leq(4 C(m+1))^{-2^{m}}}\left|P_{k}(z+y)\right| \leq(C(m+1))^{2^{k} / 4}\left(\frac{3 m}{(4 C(m+1))^{2^{m}}}\right)^{2^{k-m}}< \\
& \left.\frac{(3 m)^{2^{k-m}}}{(4 C(m+1))^{2^{k-1}}}<\left(\frac{3 m}{4 C(m+1)}\right)\right)^{2^{k-1}}<\left(\frac{3}{4}\right)^{2^{k-1}}<2^{-k}
\end{aligned}
$$

if also $k>5$, because we may assume that $C>1$.

Thus $f(z)=\sum_{k=1}^{\infty} c_{k} P_{k}(z)$ defines a holomorphic function for every sequence $\left(c_{k}\right)$ of complex numbers such that $\left|c_{k}\right| \leq k$, see [3, 2.4 and 2.8]. From (5) we deduce there exists a subsequence $\left(n_{k}\right)$ in $\mathrm{N}$ such that $\left|P_{n_{j}}\left(a_{n_{k}}\right)\right| \leq 2^{-j}$ when $j>k$. But then, since $P_{k}\left(a_{k}\right)=1$ for every $k$, for a suitable choice of signs of $c_{k}$, where $\left|c_{k}\right|=k$, we have that $\left|f\left(a_{n_{k}}\right)\right|>k-\sum_{j>k} j 2^{-j} \rightarrow \infty$, as $k \rightarrow \infty$, where $f(z)=\sum_{k=1}^{\infty} c_{k} P_{n_{k}}(z)$. Hence $D$ is not bounding and the Theorem is proved.

Definition 3. Let $r B_{E}$ denote the open ball of $E$ centered at origo and with radius $r$. A set $D \subset r B_{E}$ is called $H(r B)$-bounding if every function holomorphic on $r B_{E}$ is bounded on $D$.

A set $D$ is called strongly bounding (the class $\mathscr{B}_{s}$ from the introuction), if $D$ is $H(r B)$-bounding for every $r>\sup _{z \in D}\|z\|$.

REMARK 6. The notion of strongly bounding sets was introduced in [5]. In [5] it is shown that every limited set in $l^{\infty}$ is strongly bounding. Moreover it is proved that a set $D \subset l^{\infty}$ is limited if and only if $D$ does not contain any isomorphic copy of the unit vectors of $l_{1}$. Thus, according to the Theorem below, every limited set in $l^{\infty}$ is limited in the strongest sense. Especially this holds for the unit vectors of $c_{0}$ or more generally the unit ball of $c_{0}$ and we may take $C=1$.

THEOREM 3. Strongly bounding sets in Banach spaces are limited in the strongest sense with constant $C=1 / \sup _{z \in D}\|z\|$. More precisely, if $D \subset E$ is $H\left(r B_{E}\right)$-bounding then $D$ is limited in the strongest sense with constant $C=1 / r$.

Proof. It is enough to prove the second statement. Assume that there is $\left(\varphi_{k}\right) \subset E^{\star}$ such that

$$
\limsup _{k \rightarrow \infty} \sup _{z \in D}\left|\varphi_{k}(z)\right|=1
$$

but $\sup _{\|z\|=1} \lim \sup _{k \rightarrow \infty}\left|\varphi_{k}(z)\right|<1 / r$. Then

$$
f(z)=\sum_{k=1}^{\infty} c_{k} \varphi_{k}^{k}(z)
$$

is a holomorphic function on $r B_{E}$ for every choice of $c_{k} \in \mathrm{C}$ such that 


$$
\limsup _{k \rightarrow \infty}\left|c_{k}\right|^{1 / k} \leq 1
$$

Put $K=\sup _{z \in D}\|z\| / r<1$. Hence $\limsup _{k \rightarrow \infty}\left|\varphi_{k}(z)\right|<K$ for every $z \in D$. Thus there exist sequences $\left(a_{k}\right) \subset D$ and $\left(c_{k}\right) \subset \mathrm{C}$ such that

$$
\limsup _{k \rightarrow \infty}\left|c_{k}\right|^{1 / k} \leq 1
$$

and such that $\limsup _{k \rightarrow \infty}\left|c_{k} \varphi_{k}^{k}\left(a_{k}\right)\right| \geq k$ but $\left|c_{k} \varphi_{k}^{k}\left(a_{j}\right)\right| \leq K^{k}$ for every $k$ and $j<k$. But then a Cauchy-estimate gives that there exists a choice of $\left(c_{k}\right)$ such that $\left|f\left(a_{k}\right)\right| \rightarrow \infty$ as $k \rightarrow \infty$. This contradicts the fact that $D$ is $H(r B)$ bounding and the Theorem is proved.

REMARK 7. Example 2 shows that there are limited sets in the strongest sense which are not even bounding. A partial converse holds.

Definition 4. An entire function $f: E \rightarrow C$ is said to be generated by linear functionals if $f(z)=\sum_{k=0}^{\infty} \prod_{m=1}^{k} \varphi_{(m, k)}(z)$, where $\varphi_{(m, k)} \in E^{\star}$.

Moreover $f$ is said to be generated by uniformly bounded linear functionals if in addition

$$
\sup _{k} \prod_{m=1}^{k}\left\|\varphi_{(m, k)}\right\|^{\frac{1}{k}}
$$

is finite.

The class of sets which are bounding for entire functions generated by linear functionals is denoted $\mathscr{B}_{l}$ and the class of sets bounding for uniformly bounded linear functionals is denoted $\mathscr{B}_{l c}$.

REMARK 8. If $f(z)=\sum_{k=0}^{\infty} \prod_{m=1}^{k} \varphi_{(m, k)}(z)$ is an entire function it is easy to see that $\prod_{m=1}^{k}\left\|\varphi_{(m, k)}\right\|^{\frac{1}{k}}<C^{k} k^{k}$, for some constant $C$ independent of $k$. This estimate is also the best possible as Example 2 shows. Note also that the entire functions in Example 2 and 3 are generated by linear functionals. Thus the class $\mathscr{B}_{l}$ neither is closed under taking convex hulls nor has the G-property.

THEOREM 4. Strongly uniformly limited sets are bounding for the class of holomorphic functions generated by uniformly bounded linear functionals.

Proof. Let $D \subset E$ be a non-bounding set for the class $\mathscr{B}_{l c}$. Then there exist a sequence $\left(a_{k}\right) \subset D$, a constant $K>0$ and an entire function $\left.f(z)=\sum_{k=1}^{\infty} P_{k}(z)\right)=\sum_{k=1}^{\infty} \prod_{m=1}^{t_{k}} \psi_{m, k}(z)$, where $\psi_{m, k} \in E^{\star}$ and $\left\|\psi_{m, k}\right\|<K$, such that $P_{k}\left(a_{k}\right)>1$. Thus we may, for each $k$, rearrange and multiply $\psi_{m}^{(k)}$ with suitable constants such that $P_{k}(z)=\prod_{m=1}^{t_{k}} \varphi_{m, k}(z)$ where $\varphi_{m, k} \in E^{\star}$ is such that $\varphi_{m, k}\left(a_{k}\right)>1$ and such that $\left\|\varphi_{m-1, k}\right\| \leq\left\|\varphi_{m, k}\right\|$ for every $k$ and $m$. It 
is no loss of generality to assume that $\left\|a_{k}\right\| \leq 1$, for every $k$, and hence $\left\|\varphi_{m, k}\right\|>1$ for every $k$ and $m$.

Assume now that $D$ is strongly uniformly limited. Then $\left(a_{k}\right)$ has a subsequence which is limited in the strongest sense. We also denote this subsequence by $\left(a_{k}\right)$ and we let $f$ be defined by the corresponding subsequence of $\left(P_{k}\right)$. (Below we shall frequently take subsequences of $\left(a_{k}\right)_{k}$ and then define $f$ by the corresponding subsequence of $\left(P_{k}\right)_{k}$.) Let $C<1$ be a constant, as in Definition 1, belonging to $\left(a_{k}\right)$ and write

$$
P_{k}=\prod_{n} \prod_{m=v_{(n, k)}}^{v_{(n+1, k)}-1} \varphi_{m, k}
$$

where $v_{(n, k)} \in\{1,, k\}$ is defined by $m \geq v_{(n, k)} \Leftrightarrow\left\|\varphi_{m, k}\right\| \geq K^{n}$, which is welldefined since $1 \leq\left\|\varphi_{m-1, k}\right\| \leq\left\|\varphi_{m, k}\right\|$. Thus $v_{(0, k)}=1$ and, if $v_{(n, k)}<v_{(n+1, k)}$, we have that $K^{n} \leq\left\|\varphi_{m, k}\right\|<K^{n+1}$ when $v_{(n, k)} \leq m<v_{(n+1, k)}$. Note that $\prod_{m=1}^{t_{k}}\left\|\varphi_{m, k}\right\|<K^{t_{k}}$ because each $\left\|\psi_{m, k}\right\|<K$. Hence $K^{\sum_{n} n u_{(n+1, k)}}<K^{t_{k}}$, where $u_{(n+1, k)}=v_{(n+1, k)}-v_{(n, k)}$ if $v_{(n+1, k)}$ is defined and $u_{(n+1, k)}=t_{k}+1-v_{(n, k)}$ if $v_{(n+1, k)}$ is not defined (there is no $m$ with $K^{n+1} \leq\left\|\varphi_{m, k}\right\|$ ). Thus $\sum_{n=0}^{\infty} \frac{n u_{(n+1, k)}}{t_{k}} \leq 1$. By passing to subsequences of subsequences and finally to a diagonal sequence (we define $f$ by the corresponding subsequences of $\left(P_{k}\right)$ ), if necessary, we may assume that $\lim _{k \rightarrow \infty} \frac{u_{(n, k)}}{t_{k}}=u_{n}$ exists for every $n$ and that $\left|\frac{u_{(n, k)}}{t_{k}}-u_{n}\right|<1 / k^{2}$ for every $n \leq k$. Note also that $\sum_{n} u_{n}=1$ because $\left(t_{k}-v_{(k, k)}\right) k<t_{k} \Rightarrow\left(t_{k}-v_{(k, k)}\right) / t_{k}<1 / k$.

We now fix $n$. Put $\psi_{k, 1}=\frac{1}{u_{(n, k)}} \sum \varphi_{m, k}$, where the sum is over all $m$ such that $K^{n-1} \leq\left\|\varphi_{m, k}\right\|<K^{n}$. (If $u_{(n, k)}=0$ we put $\psi_{k, 1}=0$ ). Since $\psi_{k, 1}\left(a_{k}\right)>1$ and since $\left(a_{k}\right)$ is limited in the strongest sense, there exists $y_{1} \in E$ such that $\left\|y_{1}\right\|=1$ and

$$
\limsup _{k \rightarrow \infty}\left|\psi_{k, 1}\left(y_{1}\right)\right|>C \text {. }
$$

Hence there are infinitely many $k$ such that for at least $r_{(1, k)}=\frac{u_{(n, k)} C}{2 K^{n}}$ different $m$, we have that $\left|\varphi_{m, k}\left(y_{1}\right)\right|>C / 2$. After passing to a subsequence, if necessary, (of $\left(a_{k}\right)$ and $\left(P_{k}\right)$ ) we may assume that $r_{(1, k)}=\frac{u_{(n, k)} C}{2 K^{n}}$ exist as above for every $k \geq 1$. Put $\psi_{k, 2}=\frac{1}{u_{(n, k)}-r_{(1, k)}} \sum \varphi_{m, k}$ where the sum is over the $u_{(n, k)}-r_{(1, k)}$ different $m$ containing all the $m$ such that $\left|\varphi_{m, k}\left(y_{1}\right)\right| \leq C / 2$. Then

$$
\psi_{k, 2}\left(a_{k}\right)=\frac{1}{u_{(n, k)}-r_{(1, k)}} \sum \varphi_{m, k}\left(a_{k}\right)>1
$$

Thus, applying the limitedness in the strongest sense again, we can pick $y_{2} \in E$ such that $\left\|y_{2}\right\|=1$ and such that limsup $\left|\psi_{k, 2}\left(y_{2}\right)\right|>C$. Hence there are infinitely many $k$ such that for at least 


$$
\left.r_{(2, k)}=\frac{\left(u_{(n, k)}-r_{(1, k)}\right) C}{2 K^{n}}=p_{n}\left(1-p_{n}\right) u_{(n, k)}\right)
$$

different $m$, we have that $\left|\varphi_{m, k}\left(y_{2}\right)\right|>C / 2$, where $p_{n}=1-\frac{C}{2 K^{n}}$. Thus, after passing to a subsequence if necessary, we may assume that, for every $k \geq 2$, at least $r_{(2, k)}=\frac{\left(u_{(n, k)}-r_{(1, k)}\right) C}{2 K^{n}}$ different $m$ satisfy $\left|\varphi_{m, k}\left(y_{2}\right)\right|>C / 2$. Now we can proceed in this manner and get a diagonal sequence such that, for every $s$, there exists $y_{s} \in E$ with $\left\|y_{s}\right\|=1$, such that for $k \geq s$, at least $r_{(s, k)}=$ $p_{n}^{s-1}\left(1-p_{n}\right) u_{(n, k)}$ different $m$ satisfy $\left|\varphi_{m, k}\left(y_{s}\right)\right|>C / 2$. Put $h_{(n, k)}=u_{(n, k)}-$ $\sum_{s=1}^{k} r_{(s, k)}=u_{(n, k)}\left(1-\left(1-p_{n}\right) \sum_{s=1}^{k} p_{n}^{s-1}\right)=u_{(n, k)} p_{n}^{k} \quad$ and $\quad q_{(n, k)}=h_{(n, k)} / t_{k}$. Note that $\lim _{k \rightarrow \infty} q_{(n, k)}=0$.

Put $Q_{n, k}=\prod_{m=v_{(n-1, k)}}^{v_{(n, k)}-1} \varphi_{m, k}$ and put $z_{(n, k)}=\sum_{s=1}^{\infty} e^{i \Theta_{(s, k)}}\left(1-p_{n}\right) p_{n}^{s} y_{s}$, where $\Theta_{(s, k)} \in[0,2 \pi]$. Note that $\sum_{n=0}^{\infty}\left(1-p_{n}\right) p_{n}^{s}=1$. Then a Cauchy estimate gives that there exist a choice of $\theta_{(s, k)}$ such that, for every $k$,

$$
\begin{gathered}
\sup _{|t|=r}\left|Q_{n, k}\left(z_{(n, k)}+t a_{k}\right)\right|^{\frac{1}{t_{k}}}>\prod_{s=1}^{k}\left(\left(1-p_{n}\right) p_{n}^{s} C / 2\right)^{r_{(s, k)} / t_{k}} r^{q_{(n, k)}}= \\
\prod_{s=1}^{k}\left(\left(1-p_{n}\right) p_{n}^{s} C / 2\right)^{\frac{u_{(n, k)} t_{k}}{t_{n}^{s}\left(1-p_{n}\right)} r^{q_{(n, k)}}=} \\
\left(\frac{C}{2}\right)^{u_{(n, k)} / t_{k}} e^{-\sum_{s=1}^{k} \ln \left(\left(1-p_{n}\right) p_{n}^{s}\right) p_{n}^{s}\left(1-p_{n}\right) u_{(n, k)} / t_{k}} r^{q_{(n, k)}}> \\
\left(\frac{C}{2}\right)^{u_{n}+1 / k^{2}} e^{-T_{n}\left(u_{n}+1 / k^{2}\right)} r^{q_{(n, k)}}
\end{gathered}
$$

where $T_{n}=\sum_{s=1}^{\infty} 2 \ln \left(\left(1-p_{n}\right) p_{n}^{s}\right) p_{n}^{s}\left(1-p_{n}\right)$. An integral estimate shows that $\sum_{s} \ln \left(\left(1-p_{n}\right) p_{n}^{s}\right) p_{n}^{s}\left(1-p_{n}\right)<T n$ where $T$ just depends on $K$ and $C$.

Once again for a suitable diagonal sequence, also denoted $\left(a_{k}\right)$ with the corresponding $\left(P_{k}\right)$, we get that $\Theta_{s}=\lim _{k \rightarrow \infty} \Theta_{(s, k)}$ exist for every $s$. Put $z_{n}=\sum_{1}^{\infty} e^{i \theta_{s}} \frac{1}{s^{2}} y_{s}$ and $x_{(n, k)}=z_{(n, k)}-z_{n}$. Then

$$
\sup _{|t|=r}\left|Q_{n, k}\left(z_{n}+x_{(n, k)}+t a_{k}\right)\right|^{\frac{1}{t_{k}}}>\left(\frac{C}{2}\right)^{u_{n}+1 / k^{2}} e^{-T n\left(u_{n}+1 / k^{2}\right)} r^{q_{(n, k)}}
$$

where $x_{(n, k)} \rightarrow 0$ as $k \rightarrow \infty$.

Since this holds for every $n$ starting with any subsequence of $\left(a_{k}\right)$, we may inductively take a diagonal sequence, also denoted $\left(a_{k}\right)$, such that

$$
\sup _{|t|=r}\left|Q_{n, k}\left(z_{n}+x_{(n, k)}+t a_{k}\right)\right|^{\frac{1}{t_{k}}}>\left(\frac{C}{2}\right)^{u_{n}+1 / k^{2}} e^{-T n u_{n}-T / k} r^{q_{(n, k)}}
$$


holds for every $n$ and every $k \geq n$. Since $x_{(n, k)} \rightarrow 0$ as $k \rightarrow \infty$, we may also assume that $\sum_{n=1}^{k}\left\|x_{(n, k)}\right\| \rightarrow 0$ (the original sequence $\left(P_{k}\right)$ can be made as thin as wanted). Furthermore $q_{k}=\sum_{n=1}^{k} q_{(n, k)}+\left(t_{k}-v_{(k, k)}\right) / t_{k}<$ $\sum_{n=1}^{k} \frac{u_{(n, k)}}{t_{k}} p_{n}^{k}+1 / k<\sum_{n=1}^{k} u_{n} p_{n}^{k}+2 / k \rightarrow 0$ as $k \rightarrow \infty$ because $u_{(n, k)} / t_{k}<u_{n}+$ $1 / k^{2}, \sum_{n} u_{n}=1$ and $p_{n}^{k} \rightarrow 0$ as $k \rightarrow \infty$ for fixed $n$. But then the Cauchy estimate and the diagonal argument above, with $n$ corresponding to $s$, $z_{n}+x_{(n, k)}$ to $y_{s}$ and $n u_{n}$ to $\frac{1}{s^{2}}$ (note that $\sum n u_{n}$ is convergent), give that there exist a subsequence, also denoted $\left(a_{k}\right), z=\sum_{n=1}^{\infty} n u_{n} e^{i \theta_{n}^{\prime}} z_{n}$ and $x_{k}$ such that $x_{k} \rightarrow 0$ as $k \rightarrow \infty$ and such that

$$
\begin{gathered}
\sup _{|t|=r}\left|P_{k}\left(z+x_{k}+t a_{k}\right)\right|^{\frac{1}{t_{k}}}> \\
\left(\frac{C}{2}\right)^{\sum u_{n}+1 / k} e^{-\sum T n u_{n}-T} \prod\left(n u_{n}\right)^{u_{n}} r^{q_{k}}>\left(\frac{C}{2}\right)^{2} e^{-2 T} C_{1} r^{q_{k}}
\end{gathered}
$$

where the sums and the product is over all $n$ and $\prod\left(n u_{n}\right)^{u_{n}}>C_{1}>0$. But this contradicts the fact that $f$ is holomorphic on $E$, because if $R$ is so big that $R\left(\frac{C}{2}\right)^{2} e^{-T} C_{1}>1$, then $f$ is not bounded in any neighbourhood of $R z$. Hence $D$ is not strongly uniformly limited and the theorem is proved.

REMARK 9. Example 2 shows that the class $\mathscr{B}_{l_{c}}$ cannot be replaced by $\mathscr{B}_{l}$ and a close look at the example of [6] shows that strongly uniformly limited cannot be replaced by uniformly limited. The unit vectors of $c_{0}$ in [6] is in fact limited in the strong sense.

\section{REFERENCES}

1. J. Bourgain and J. Diestel, Limited operators and strict cosingularity, Math. Nachr. 119 (1984), 55-58.

2. S. Dineen, Bounding subsets of a Banach space, Math. Ann. 192 (1971), 61-70.

3. S. Dineen, Complex analysis in locally convex spaces, North-Holland Studies 57 (1981).

4. L. Drewnowski, On Banach spaces with the Gelfand-Phillips property, Math. Z. 193 (1986), 405-411.

5. B. Josefson, Bounding subsets of $l^{\infty}(A)$, J. Math. Pures Appl. 57 (1978), 397-421.

6. B. Josefson, A Banach space containing non-trivial limited sets but no non-trivial bounding sets, Israel J. Math. 71 (1990), 321-327.

7. T. Schlumprecht, A limited set that is not bounding, Proc. Roy. Irish Acad. Sect. A 90 (1990), $125-129$.

8. T. Schlumprecht, Limited sets in $C(K)$-spaces and examples concerning the Gelfand-Phillips property. Math. Nachr. 157 (1992), 51-64.

9. T. Schlumprecht, Limited sets in Banach spaces, Dissertation 1988. 